\title{
Mohamed Kamel DORAÏ, Les réfugiés palestiniens du Liban : une géographie de l'exil
}

Sari Hanafi

\section{(2) OpenEdition}

1 Journals

Édition électronique

URL : https://journals.openedition.org/remi/4191

DOI : 10.4000/remi.4191

ISSN : $1777-5418$

Éditeur

Université de Poitiers

Édition imprimée

Date de publication : 1 octobre 2007

Pagination : 218-219

ISBN : 978-2-911627-46-6

ISSN : 0765-0752

\section{Référence électronique}

Sari Hanafi, « Mohamed Kamel DORAï, Les réfugiés palestiniens du Liban : une géographie de l'exil », Revue européenne des migrations internationales [En ligne], vol. 23 - n² | 2007, mis en ligne le 27 novembre 2008, consulté le 16 avril 2022. URL : http://journals.openedition.org/remi/4191 ; DOI : https://doi.org/10.4000/remi.4191

Ce document a été généré automatiquement le 16 avril 2022.

() Université de Poitiers 


\title{
Mohamed Kamel DORAÏ, Les réfugiés palestiniens du Liban : une géographie de l'exil
}

\author{
Sari Hanafi
}

\section{RÉFÉRENCE}

Mohamed Kamel DORAII, Les réfugiés palestiniens du Liban : une géographie de l'exil, Paris : CNRS Éditions, 2006, Collection : Moyen-Orient, 256 p. Illustrations : 15 cartes N \& b, 15 tableaux, 3 figures, ISBN : 2-271-06391-4.

1 «Ce n'est pas en termes de pertes militaires, de pertes économiques [...] que l'on peut estimer de la manière la plus réaliste quel a été le prix à payer par les peuples du Proche-Orient pour les événements de l'année dernière, mais en termes de changements politiques dont le plus marquant a été la création d'une nouvelle catégorie d'apatrides, les réfugiés arabes. » C'est par cette citation de Hannah Arendt qui date de 1948 que Mohamed Kamel Doraï commence son livre pour souligner l'étendue et la gravité du problème des réfugiés palestiniens au Liban sans pour autant tomber dans la mythologie de l'unicité de ce groupe et le misérabilisme, qui ont souvent caractérisé les travaux sur les réfugiés palestiniens.

2 L'auteur a étudié l'espace des camps des réfugiés au Liban et les enjeux liés à sa maîtrise, à ses aménagements ainsi qu'à la symbolique qui s'y rattache. C'est un regard de géographe et d'anthropologue que porte l'auteur qui a lui-même vécu dans le camp qu'il étudie. Il apporte un éclairage nouveau sur la question des réfugiés en replaçant les mutations de l'espace dans lequel ils s'inscrivent au cœur des problématiques régionales et notamment celle du contrôle de l'espace au centre des tensions moyenorientales.

3 Kamel Doraï souligne une singularité des réfugiés palestiniens au Liban par rapport à ceux qui vivent en Syrie et en Jordanie. Alors que l'Autorité libanaise les considère 
comme étrangers, l'accès au marché de l'emploi leur est quasiment interdit et tout particulièrement pour les professions libérales, ce qui entraîne une marginalité économique remarquable. Cependant les différentes études de cas menées par l'auteur permettent de mettre en évidence des réalités très contrastées et montrent une capacité de transgression comme au Camp d'Al Buss par exemple qui a su se connecter au tissu urbain de la ville de Tyr.

On relèvera dans l'ouvrage la référence à l'histoire pour appréhender la discrimination systématique contre les réfugiés. Il a fallu dix ans pour que les tentes disparaissent et que l'habitat en dur apparaisse. Mais l'autorité sécuritaire du Liban qui était responsable des camps avec l'aide de l'UNRWA a refusé pendant longtemps que les réfugiés remplacent les toits en zinc par du béton et encore aujourd'hui les traces de zinc sont visibles dans différents camps comme Al Badawi et Ain Al Helweh.

5 Mais l'originalité de ce livre repose sur des données originales exploitées afin d'analyser finement l'espace des camps qui, pour l'auteur, remplit quatre fonctions principales: un lieu d'habitat, un espace de développement ou de repli économique pour les réfugiés, un lieu de mémoire et d'affirmation identitaire ainsi qu'un lieu d'exercice et de développement du pouvoir politique et militaire. Ces différentes fonctions créent une dynamique socio-spatiale palestinienne qui repose sur trois aspects : la permanence territoriale (un lieu de stabilité et de continuité), le lieu de «l'entre-soi » et l'espace de contact avec la société libanaise. Sur ce dernier point, Kamel Doraï montre que le camp d'Al Buss a pu établir des contacts et s'intégrer dans le tissu urbain, ce qui n'est pas le cas d'autres camps.

6 Tourné vers une construction identitaire où la mémoire joue un rôle important, mais tourné aussi vers la société d'accueil si problématique que soit ce contact, le camp comme espace vivant est un "véritable laboratoire pour la société palestinienne à venir ", selon Mohamed Kamel Doraï. Mais, le camp est aussi un laboratoire de contrôle et de surveillance avec la présence de différentes autorités, qu'elles soient celles d'accueil ou celles des fractions politiques palestiniennes. De ce point de vue, on peut regretter que l'auteur n'ait pas suffisamment analysé le camp comme un espace disciplinaire où le pouvoir coercitif, même à l'intérieur du camp, a beaucoup pesé sur les habitants. L'« espace camp » se présente comme des lieux hétérotopiques, au sens de Michel Foucault, non seulement déconnectés du tissu social et urbain qui les entoure, comme le souligne kamel Doraï, mais aussi comme un espace de tensions, de marginalité et de contradiction ; à la fois espace de contrôle et de marge il favorise des actes potentiels de résistance et de transgression. L'apogée de cette situation s'incarne dans la présence du groupe radical Arabe proche d'Al-Qaida dans le camp de Nahr Al Bared.

7 La situation socio-économique et spatiale des Palestiniens réfugiés et la guerre civile libanaise a conduit à l'exil selon l'auteur environ 100000 Palestiniens qui ont quitté le Liban pour se diriger vers des pays européens, en particulier les pays scandinaves et l'Allemagne. Kamel Doraï décrit ces communautés dans leur exil, leur dispersion et leur accès à une nationalité européenne ou nord-américaine. Alors que circulait, tant dans l'imaginaire populaire que dans la communauté scientifique le mythe de l'effritement du sentiment de l'appartenance à la Palestine ou l'idée que le droit au retour des réfugiés palestiniens serait remis en question avec l'acquisition d'une nationalité, l'auteur au fil de sa démonstration conteste cette corrélation. De nombreux exemples 
montrent en effet que le sentiment d'appartenance peut perdurer, même lorsque des groupes sont considérés comme totalement « intégrés » à leur société d'accueil.

8 Kamel Doraï conclut que les développements en Territoire palestinien ainsi que le processus de paix, même avorté, dans la recherche d'une création d'une "entité " palestinienne, ont contribué à la modification de la géographie de la diaspora. L'implication spatiale de cette redéfinition est double: les camps de réfugiés et leurs habitants sont de plus en plus isolés au sein de l'organisation de l'espace diasporique palestinien, et des dynamiques migratoires se développent qui échappent aujourd'hui aux logiques de l'OLP et des institutions diasporiques. D'où les nouveaux clivages entre l'intérieur (le Territoire palestinien) et l'extérieur (les militants actifs des camps de réfugiés). Mais ce que Kamel Doraï n'a peut-être pas osé dire est que l'on assiste à une fragmentation du nationalisme palestinien entre un versant radical des camps de réfugiés (qui supporte les groupes radicaux comme le Hamas) et un versant proche de l'OLP, en faveur d'un compromis territorial et d'un compromis sur les modalités d'application du droit de retour.

9 Finalement, la lecture du livre de Mohamed Kamel Doraï s'avère indispensable non seulement pour comprendre la communauté palestinienne du Liban mais aussi pour comprendre l'effet de la mondialisation forcée sur la dynamique socio-politique dans la région du Moyen-Orient.

\section{AUTEURS}

\section{SARI HANAFI}

Professeur associé, American University of Beirut 